\title{
CRISPR/Cas9-mediated targeted mutagenesis of GmLHY genes alters plant height and internode length in soybean
}

Qun Cheng ${ }^{1 \dagger}$, Lidong Dong ${ }^{1 \dagger}$, Tong Su${ }^{2,3+}$, Tingyu Li ${ }^{1 \dagger}$, Zhuoran Gan ${ }^{1}$, Haiyang Nan', Sijia Lu', Chao Fang ${ }^{1}$, Lingping Kong ${ }^{1}$, Haiyang Li ${ }^{1}$, Zhihong Hou' ${ }^{1}$ Kun Kou ${ }^{2,3}$, Yang Tang ${ }^{1}$, Xiaoya Lin ${ }^{1}$, Xiaohui Zhao ${ }^{1}$, Liyu Chen ${ }^{1}$, Baohui Liu ${ }^{1,2^{*}}$ and Fanjiang Kong ${ }^{1,2^{*}}$ (i)

\begin{abstract}
Background: Soybean (Glycine max) is an economically important oil and protein crop. Plant height is a key trait that significantly impacts the yield of soybean; however, research on the molecular mechanisms associated with soybean plant height is lacking. The CRISPR (clustered regularly interspaced short palindromic repeat)/Cas9 (CRISPRassociated system 9) system is a recently developed technology for gene editing that has been utilized to edit the genomes of crop plants.

Results: Here, we designed four gRNAs to mutate four LATE ELONGATED HYPOCOTYL (LHY) genes in soybean. In order to test whether the gRNAs could perform properly in transgenic soybean plants, we first tested the CRISPR construct in transgenic soybean hairy roots using Agrobacterium rhizogenes strain K599. Once confirmed, we performed stable soybean transformation and obtained 19 independent transgenic soybean plants. Subsequently, we obtained one $T_{1}$ transgene-free homozygous quadruple mutant of GmLHY by self-crossing. The phenotypes of the $T_{2}$-generation transgene-free quadruple mutant plants were observed, and the results showed that the quadruple mutant of GmLHY displayed reduced plant height and shortened internodes. The levels of endogenous gibberellic acid (GA3) in Gmlhyla1b2a2b was lower than in the wild type (WT), and the shortened internode phenotype could be rescued by treatment with exogenous GA3. In addition, the relative expression levels of GA metabolic pathway genes in the quadruple mutant of GmLHY were significantly decreased in comparison to the WT. These results suggest that GmLHY encodes an MYB transcription factor that affects plant height through mediating the GA pathway in soybean. We also developed genetic markers for identifying mutants for application in breeding studies.
\end{abstract}

Conclusions: Our results indicate that CRISPR/Cas9-mediated targeted mutagenesis of four GmLHY genes reduces soybean plant height and shortens internodes from 20 to 35 days after emergence (DAE). These findings provide insight into the mechanisms underlying plant height regulatory networks in soybean.

Keywords: CRISPR/Cas9, Plant height, Soybean, LHY, Transgene-free

\footnotetext{
* Correspondence: liubh@iga.ac.cn; kongfj@gzhu.edu.cn

${ }^{\dagger}$ Qun Cheng, Lidong Dong, Tong Su and Tingyu Li contributed equally to this work.

${ }^{1}$ School of Life Sciences, Guangzhou University, Guangzhou, China

Full list of author information is available at the end of the article
}

(c) The Author(s). 2019 Open Access This article is distributed under the terms of the Creative Commons Attribution 4.0 International License (http://creativecommons.org/licenses/by/4.0/), which permits unrestricted use, distribution, and reproduction in any medium, provided you give appropriate credit to the original author(s) and the source, provide a link to the Creative Commons license, and indicate if changes were made. The Creative Commons Public Domain Dedication waiver (http://creativecommons.org/publicdomain/zero/1.0/) applies to the data made available in this article, unless otherwise stated. 


\section{Background}

Soybean is one of the most important economic sources of vegetable oil and protein worldwide, and plant height, node number, internode length, branch number, and seed size are significant factors that affect soybean yield $[1,2]$. Plant height is a key trait of plant ideotypes, and a relatively shorter stem length contributes to increased yield in modern breeding programs [3-5]. Some plant height genes have thus been cloned by map-based cloning in several plant species, such as maize [6-8], rice [9-11], tomato $[12]$, and soybean $[13,14]$. For example, GA3 $b$ hydroxylase (ZmGA3ox2) was cloned using candidate gene association mapping and a genetic assay from the dwarf mutant d1-6016 and responded for the dwarf mutant in maize [7]. The Brachytic2 ( $\mathrm{Br} 2)$ gene, which was cloned from maize by mapping, significantly impacts plant height [8]. Recent research showed that GmDW1 (dwarf mutant) encodes an ent-kaurene synthase, and the mutant of GmDW1 displayed reduced plant height and shortened internodes in soybean [13]. In addition, several transcription factor (TF) families play important roles in plant height. For instance, OsNAC2 is a NAC transcription factor, and the constitutive expression of OsNAC2 resulted in shorter internodes and shorter spikelets in rice [15].

Circadian clocks are endogenous 24-h oscillators that allow organisms to anticipate daily changes in their environment, playing critical roles in many biological processes and stress responses by regulating up to $80 \%$ of the transcriptome in plants [16-18]. LHY and CCA1 are key components of the central oscillator and encode two morningexpressed MYB TFs in Arabidopsis [19, 20]. AtLHY/ CCA1 can bind to the evening element (EE; AAATATCT) of the promoter of TIMING OF CAB EXPRESSION 1 (TOC1) and act redundantly to repress the transcription of the AtTOC1 gene during the day [21]. AtTOC1 represses $A t C C A 1$ and $A t L H Y$ from its induction at dusk until slightly before dawn [22]. Other functions of LHY/ CCA1 in flowering and the stress response have been reported [23, 24]. For example, silencing of NaLHY abolished the vertical movement of flowers under continuous light conditions in Nicotiana [23]. A recent report showed that AtLHY can regulate the expression of abscisic acid (ABA) signaling components and downstream response genes to potentiate some ABA responses [24]. However, the potential functions of the $L H Y / C C A 1$ family members in soybean are still unclear.

The CRISPR/Cas9 system was recently engineered for the genetic manipulation of plants [25-28]. The use of CRISPR/Cas9 technology has attracted great attention and has been successfully applied in various crops for genome editing, such as wheat [29, 30], maize [31, 32], rice [33], barley [34], tomato [35, 36], and soybean [3739]. There are four $G m L H Y$ genes in soybean, named GmLHY1a, GmLHY1b, GmLHY2a, and GmLHY2b, but the functions of these genes remain unknown. Therefore, in the current study, the CRISPR/Cas9 system was used to target four GmLHY genes in soybean. We observed the phenotype of the $\mathrm{T}_{2}$-generation transgenefree quadruple mutant of $G m L H Y$ and found that the height and internodes of the quadruple mutant were significantly shorter than that of the WT. Moreover, the relative expression levels of GA metabolic pathway genes in the quadruple mutant of $G m L H Y$ were significantly lower than in WT. These results suggested that GmLHY directly or indirectly regulates plant height by mediating key components of the GA pathway. We also developed genetic markers for the identification of mutants for use in breeding studies. Our findings suggest that the manipulation of these genes should facilitate improvements in plant height and internodes in soybean.

\section{Results}

Target site selection, construction, and confirmation of the target sites in soybean hairy roots

In order to identify the ortholog of AtLHY and AtCCA1 in soybean, we performed protein sequence alignment and identified four CCA1/LHY orthologs in soybean. Phylogenetic analysis showed that the four CCA1/LHY orthologs are closer to AtLHY than AtCCA1. Thus, the four CCA1/LHY orthologs was named GmLHY1a (Glyma.16G017400), GmLHY1b (Glyma.07G048500), GmLHY2a (Glyma.19G260900), and GmLHY2b (Glyma.03G261800) (Additional file 1: Fig. S1). To study the function of the four GmLHY genes in soybean, four target adaptors were used, including target $1 / 2$ for targeting the GmLHY2a and GmLHY2b genes, and target 3/4 for targeting the GmLHY1a and GmLHY1b genes (Fig.1a). Target 1 is present in the second and third exon of the GmLHY2b and GmLHY2a genes, respectively; target 2 is present in the fifth and sixth exon of the GmLHY2b and GmLHY2a genes, respectively; target 3 is present in the first exon of $G m L H Y 1 a$ and $G m L H Y 1 b$; and target 4 is present in the fifth exon of GmLHY1a and GmLHY1b in soybean (Fig. 1a). The CRISPR vector encodes Cas 9 and was driven by the CaMV35S promoter and four gRNAs driven by the Arabidopsis U3b, U3d, U6-1, and U6-29 promoters, respectively (Fig. 1b, c).

In order to test whether the CRISPR/Cas9 construct could properly edit these genes in transgenic soybean plants, we first tested the construct in transgenic soybean hairy roots using A. rhizogenes K599 (Additional file 1: Fig. S2A). The transgenic soybean hairy roots were generated by high-efficiency Agrobacterium rhizogenesmediated transformation [40]. When the hairy roots generated at the infection site were approximately $2 \mathrm{~cm}$ long, they were used for genotype detection. The genotype of the transgenic hairy roots was detected by PCR using Cas 9 gene-specific primers and $G m L H Y$ genespecific primers. We detected mobility-shifted bands in six DNA-bulked samples when the Cas9 gene-specific 

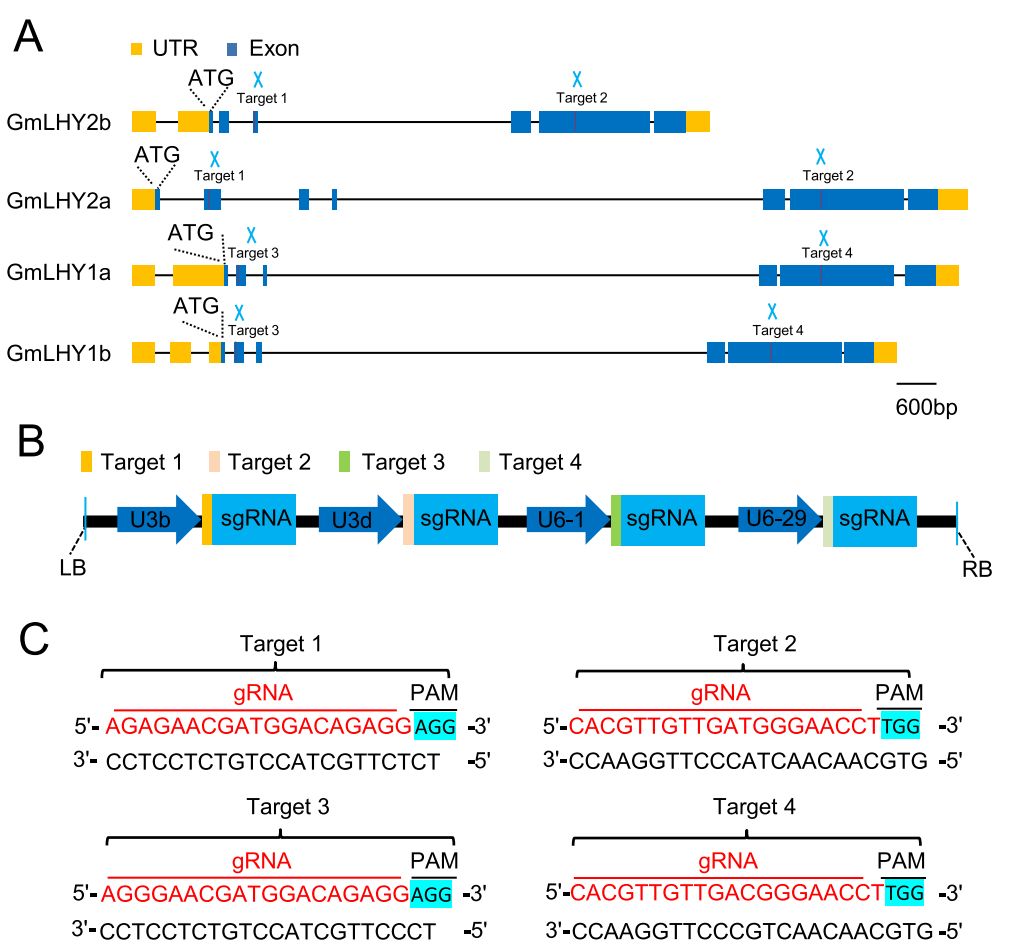

Fig. 1 Diagram of the dual gRNA CRISPR/Cas9 vector, target sequences, and target locations of the four GmLHY genes. a Location of the dual target sites in GmLHY1a, GmLHY1b, GmLHY2a and GmLHY2b. Bar =600 bp; b Schematic figure of the binary vector designed for mutagenesis of the GmLHY genes using the CRISPR/Cas9 technology; c Target sequences

primers were utilized. The result showed that there were five transgenic lines with the Cas9 gene product (Cas9 gene-positive) (Additional file 1: Fig. S2B). Sequencing analysis of the $G m L H Y$ genes showed that the Cas 9 gene-positive lines (R1-R5) produced superimposed peaks in the target $1 / 3$ site, while the target 2/4 site was unchanged (Additional file 1: Figure S2C, Additional file 2: Table S1). Together, these results indicated that the transgene-encoded Cas9 and gRNAs were able to efficiently induce double-strand breaks at the target $1 / 3$ sites in the GmLHY genes.

\section{Transgene-free homozygous quadruple mutant of $G m L H Y$ in soybean}

We next performed stable soybean transformation and obtained 19 independent $\mathrm{T}_{0}$ transgenic lines with the section for the Cas 9 gene product (Cas 9 gene-positive) (Additional file 1: Fig. S3A). Sequencing analysis showed that the $\mathrm{T}_{0}-7$ line was a heterozygous quadruple mutant of $G m L H Y$ that might possess a 2-bp deletion in GmLHY2b/2a/1b-target1/3 and a 1-bp deletion in GmLHY2a-target3 (Additional file 1: Figure S3B-E; Additional file 3: Table S2). In order to use the mutants in crop breeding, we sought homozygous quadruple mutants of the GmLHY line without the transgene and screened the $T_{1}$ plants derived from the $T_{0}$ transgenic lines. Fortunately, we obtained eight $\mathrm{T}_{1}$ plants derived from $\mathrm{T}_{0}-7$ that lacked the Cas 9 gene (Fig. 2a, b), and only one line $\left(\mathrm{T}_{1}-15\right)$ was a transgene-free homozygous quadruple mutant of $G m L H Y$ (Fig. 2c-f; Additional file 3: Table S2). Sequencing analysis showed that the quadruple mutant of $G m L H Y$ had a 2-bp deletion in GmLHY2b/2a/1b-target1/3 and a 1-bp deletion in GmLHY1a-target3 (Fig. 2c-2f), resulting in frame-shift mutations in the GmLHY genes (Fig. 2g).

\section{The expression level of GmLHY in the quadruple mutant and WT}

LHY/CCA1 are key components of the circadian clock and participate in the temporal organization of biological activities and the regulation of gene expression [16, 17, 21]. Previous studies have shown that the expression level of $L H Y / C C A 1$ was much higher in the morning than in the night [21]. However, the expression pattern of $G m L H Y$ genes in the quadruple mutant of $G m L H Y$ is not known. The diurnal circadian rhythm of GmLHY gene expression in the quadruple mutant of $G m L H Y$ was analyzed by quantitative real-time PCR (qRT-PCR) under inductive long-day (LD) conditions. The result showed that GmLHY1a, GmLHY1b, GmLHY2a, and $G m L H Y 2 b$ were highly up-regulated in WT, and the highest expression was detected at $0 \mathrm{~h}$ and $24 \mathrm{~h}$ after dawn (Fig. 3a-d). However, the expression of GmLHY genes was lower in the quadruple mutant of $G m L H Y$ 


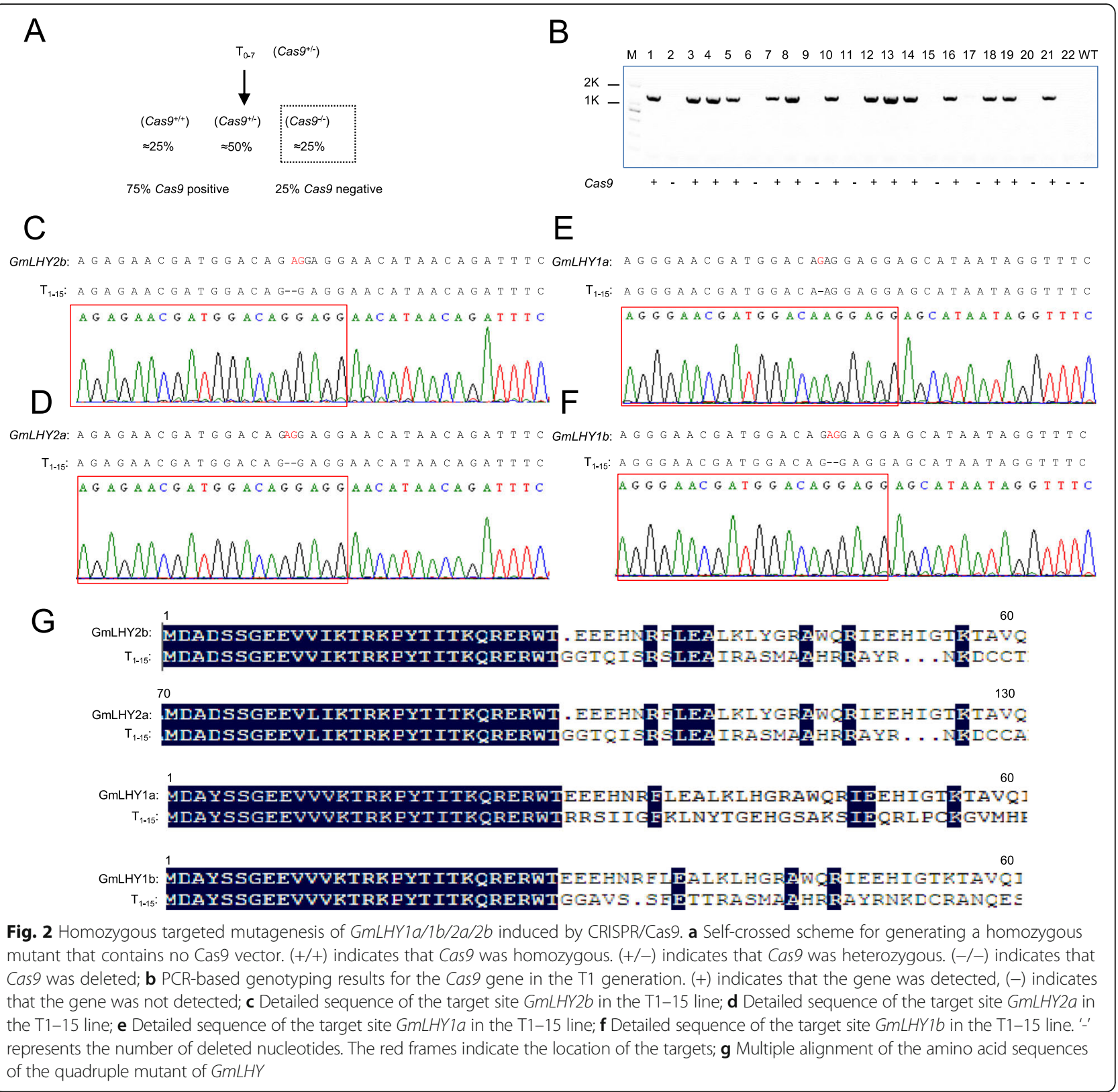

than WT (Fig. 3A-D). These results showed that the expression of the four GmLHY genes was significantly decreased in the quadruple mutant of GmLHY.

The quadruple mutant of $G m L H Y$ reduces soybean plant height and shortens internodes

To examine the loss function of $G m L H Y$, the phenotypes of the $\mathrm{T}_{2}$-generation transgene-free quadruple mutant and WT plants were observed. We found that the plant height of the quadruple mutant was significantly lower than WT under LD conditions for 20 DAE (Fig. 4a, b). Subsequently, we examined the node number and internodal length, as these impact plant height [13, 15]. As indicated in Fig. 4c and d, the node number did not change, while the internodal length was significantly shorter in the quadruple mutant than WT. These results suggested that the dwarfed plant height of the quadruple mutant was caused by a shorter length. We also analyzed the plant height of the quadruple mutant and WT from 20 to 35 DAE (Fig. 4e). The result showed that the height of the quadruple mutant of $G m L H Y$ was shorter from 20 to 35 DAE.

The quadruple mutant of $G m L H Y$ is deficient in the GA biosynthesis pathway

Previous studies showed that GAs is one of the most important phytohormones determining plant height [41, 42]. To test whether $G m L H Y$ affects the GA biosynthesis 

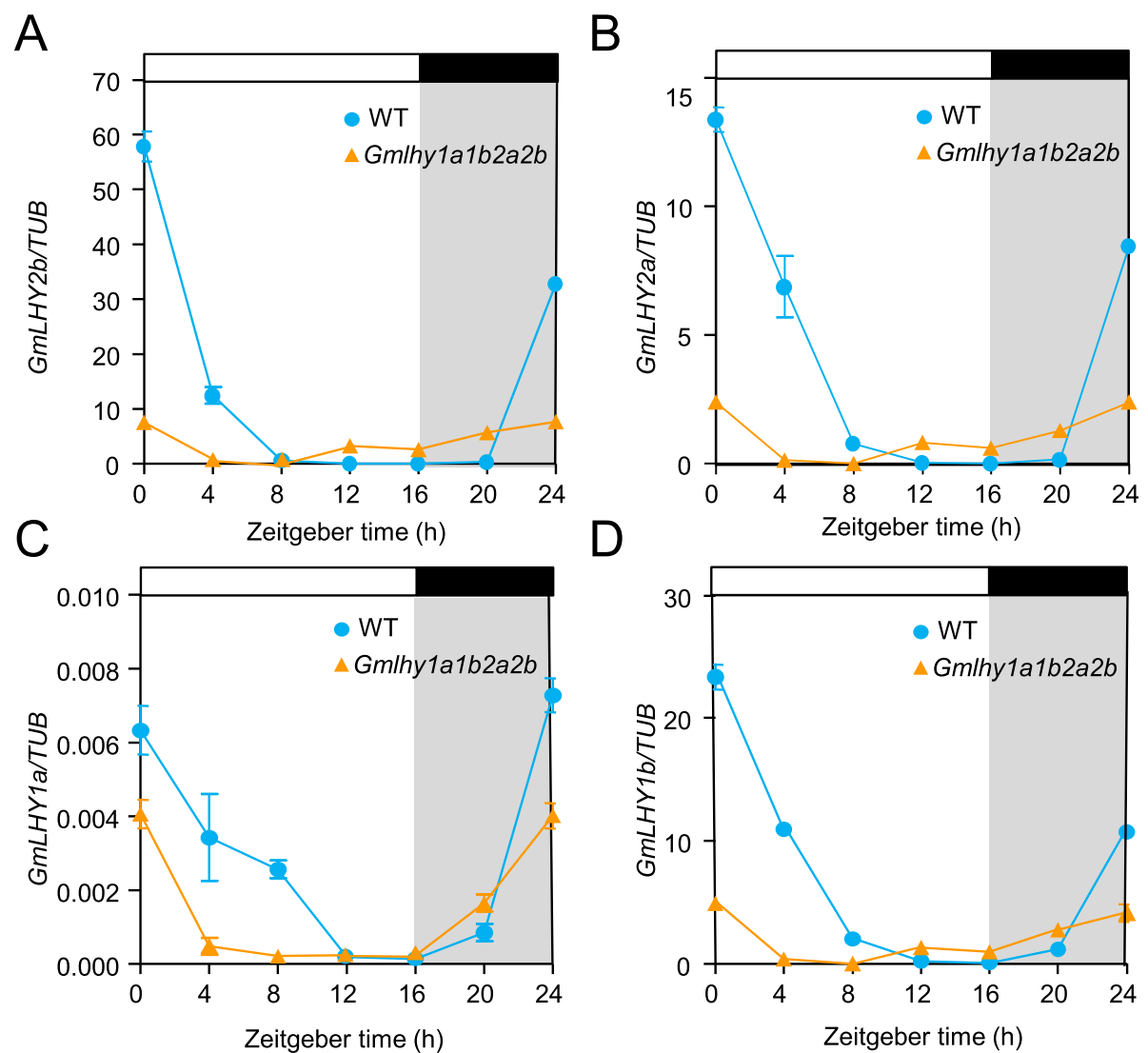

Fig. 3 Diurnal expression patterns of GmLHY1a/1b/2a/2b in WT plants and T2 homozygous quadruple mutants of GmLHY. a-d qRT-PCR analysis of GmLHY2b, GmLHY2a, GmLHY1a, and GmLHY1b expression levels in the leaves at 20 DAE under 16 h light/8 h dark (long day; LD) conditions, respectively. Data shown are relative to the control gene GmTUB and represent means \pm standard error of the mean (s.e.m.) for three biological replicates. Bars indicate the s.e.m. Black and white bars represent dark and light periods, respectively

pathway, the Gmlhy1a1b2a2b mutant and WT were treated with $\mathrm{GA}_{3}$ and Uni (uniconazole, a GA biosynthesis inhibitor). The results showed that exogenous $\mathrm{GA}_{3}$ could restore the Gmlhy1a1b2a2b mutant to the WT, and Uni treatment could reduce the plant height of the WT and Gmlhy1a1b2a2b mutant seedlings (Fig. 5a, b). Endogenous $\mathrm{GA}_{3}$ levels from both the WT and Gmlhy1a1b2a2b mutant were determined using liquid chromatography-mass spectrometry (LC-MS). The results suggested that the levels of endogenous GA3 in Gmlhy1a1b2a2b were lower than in WT (Fig. 5c). These findings indicated that the Gmlhy1a1b2a2b mutant has a low active gibberellin level and that it is a GA biosynthesis-deficient mutant.

\section{Expression analysis of GA metabolic pathway-related} genes in the quadruple mutant of GmLHY and WT plants Next, qRT-PCR was performed to measure the relative expression of genes that are known to participate in GA biosynthesis, such as GA-20 oxidase (GmGA1, Glyma.09G149200; GmGA2, Glyma.20G153400), copalyl pyrophosphate synthase (GmCPS2, Glyma.19G157000), ent-kaurene synthase (GmDW1, Glyma.08G163900), and GA-responsive genes (GmGR2, Glyma.20G230600; GmGR8, Glyma.11G216500) [13] in WT and the quadruple mutant of GmLHY. Compared with the WT plants, these genes showed significantly decreased expression in the quadruple mutant of GmLHY (Fig. 6a-f). Our findings suggested that $G m L H Y$ might positively regulate the expression of these GA biosynthesis and GA responsive genes, thereby limiting soybean plant height.

\section{Development of genetic markers and inheritance of quadruple mutant alleles}

Genetic markers provide a critical and effective means of identifying mutant alleles for molecular-assisted studies and could possibly accelerate the genotyping procedure in future generations [38]. Therefore, we developed three dCAPs (Derived Cleaved Amplified Polymorphic Sequences) markers to identify the Gmlhy1a1b2a2b mutant alleles (Fig. 7a). For the genotyping of the Gmlhy1a1b2a2b mutants, PCR amplifications were performed using GmLHY-specific and dCAPs-specific primer pairs. The amplified products of GmLHY2b, 
A

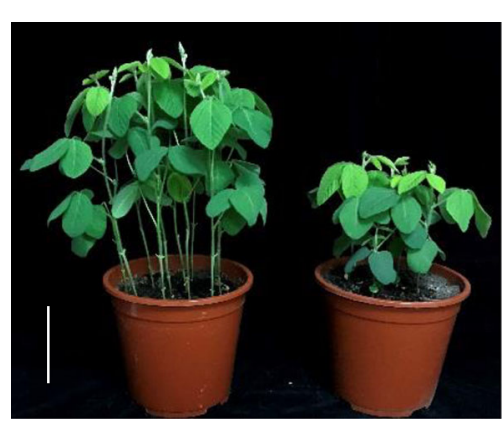

WT
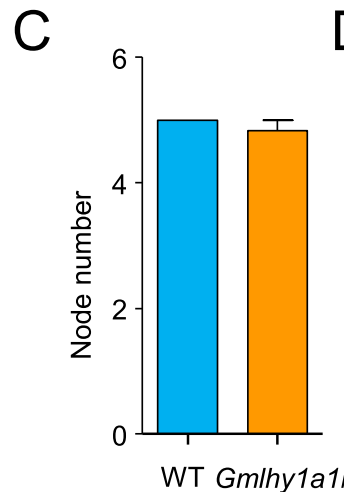

Gmlhy1a1b2a2b
B

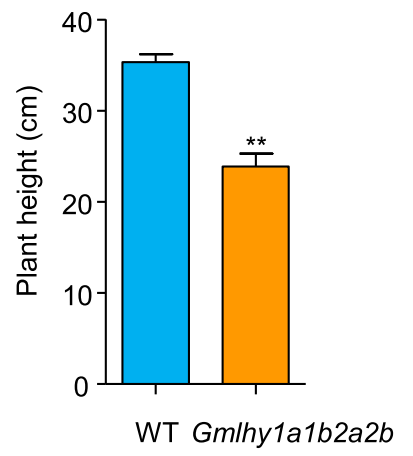

$\mathrm{E}$

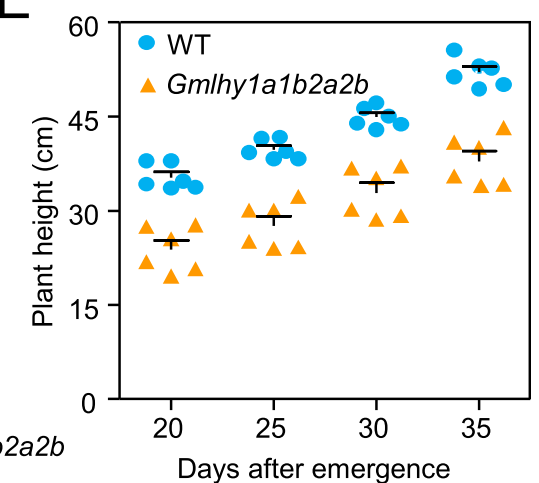

Fig. 4 The phenotype of the WT plants and T2 homozygous quadruple mutant of GmLHY. a The plant height of homozygous T2 seedings and WT; b The statistics of plant height for 20 DAE under LD conditions; $\mathbf{c}$ The statistics of node number for 20 DAE under LD conditions; $\mathbf{d}$ The statistics of intermodal length for 20 DAE under LD conditions; e The statistics of plant height from 20 to 35 DAE. The experiment was performed using six biological replicates, and differences were statistically analyzed using Student's $t$-test $\left({ }^{* *} P<0.01\right)$. Bars indicate the s.e.m. All data are shown as means \pm s.e.m. ( $n=6$ plants)

GmLHY2a, and GmLHY1b on the mutant genomic DNA templates, but not on the WT genomic DNA templates, could be cleaved by the restriction endonuclease MspI (Fig. 7b). Additionally, the amplified products of GmLHY1a on the mutant genomic DNA templates, but not on the WT genomic DNA templates, could be cleaved by restriction endonuclease RspRSII (Fig. 7b). These results confirmed that the three dCAPs markers of GmLHY could be used for the genotyping of Gmlhy1a1b2a2b mutants and have further prospect in molecular breeding studies.

\section{Discussion}

The CRISPR/Cas9 system is a recent development that has been rapidly and widely used to edit the genomes of various crops, such as soybean [37-39]. For example, Bao et al. obtained GmSPL9 gene mutants using CRISPR/Cas9 and stable soybean transformation and found that the mutant of GmSPL9s demonstrated increased node number on the main stem and branch number, resulting in increased total node number per plants [38]. The CRISPR-edited soybean plants of both the GmFAD2-1A and GmFAD2-1B genes showed dramatic increases of over $80 \%$ in oleic acid content, whereas linoleic acid content decreased to $1.3-1.7 \%$ [39]. $L H Y$ and $C C A 1$ are important circadian clock genes that encode two morning-expressed MYB TFs in Arabidopsis $[19,20]$. However, the functions of LHY/CCA1 family members in soybean are still unknown. In this study, we designed four target adaptors (target 1 , target 2, target 3 , and target 4) to edit four GmLHY genes (Fig. $1 \mathrm{a}-\mathrm{c}$ ). In order to test whether the targets could perform properly in transgenic soybean plants, we first tested the CRISPR construct in transgenic soybean hairy roots using Agrobacterium rhizogenes strain $\mathrm{K} 599$. We confirmed that target 1 and target 3 could perform, while target 2 and target 4 might not work properly (Additional file 1: Fig. S2). We then performed stable soybean transformation and obtained $19 \mathrm{~T}_{0}$ events. In previous CRISPR/Cas9 research, chimeric mutations reduced the heritable transmission of mutant alleles in soybean [43, 44]. Therefore, in this study, we sought homozygous quadruple mutants of the $G m L H Y$ lines without transgenes and screened $\mathrm{T}_{1}$ plants derived from the $T_{0}$ transgenic lines. Fortunately, we obtained one $\left(\mathrm{T}_{1}-15\right)$ transgene-free homozygous quadruple mutant of GmLHY (Fig. 2Fc-f; Additional file 


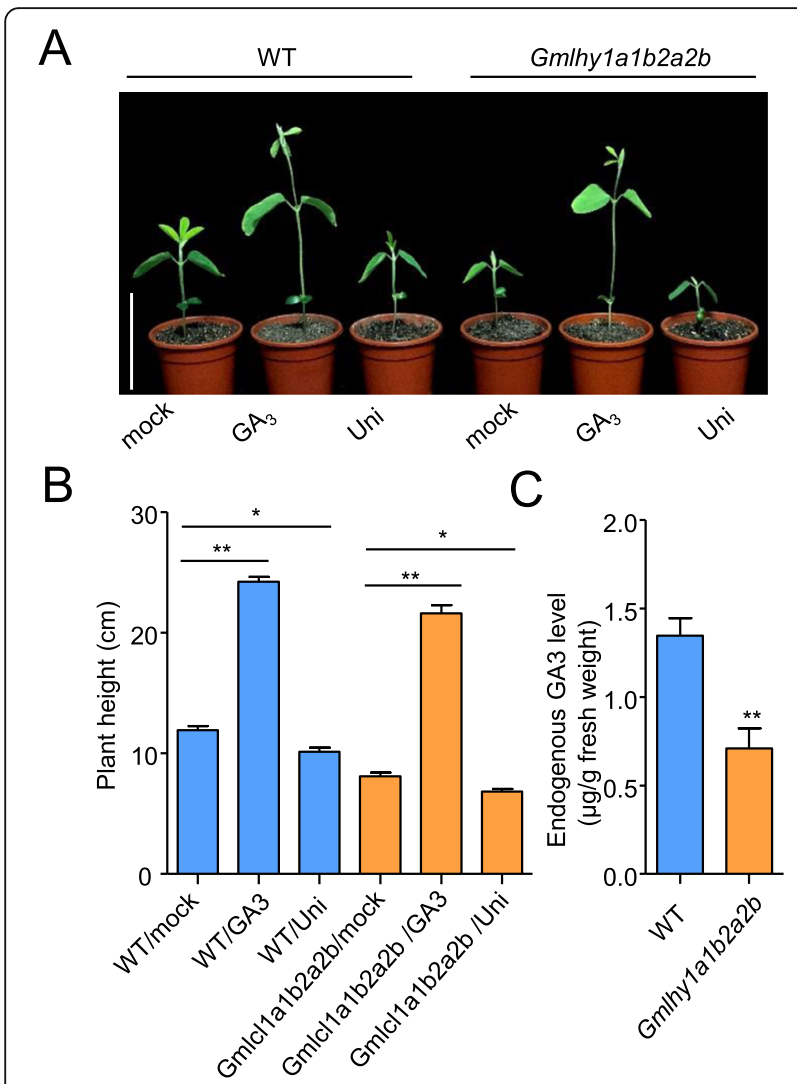

Fig. 5 The quadruple mutant of GmLHY is a GA-deficient soybean mutant. a The morphological phenotypes of WT and quadruple mutant $4 \mathrm{~d}$ past the exogenous GA3 (1 mg/L) and Uni (1 mg/L) application. $\mathbf{b}$ The statistical data of plant height of WT and quadruple mutant $4 \mathrm{~d}$ past the exogenous GA3 (1 mg/L) and Uni (1 $\mathrm{mg} / \mathrm{L}$ ) application. The experiment was performed using three biological replicates, and differences were statistically analyzed using Student's t-test $\left({ }^{*} P<0.05 ;{ }^{*} P<0.01\right)$. Bars indicate the s.e.m. $\mathbf{c}$ Determination of endogenous GA3 levels in the leaves of 20-day-old WT and quadruple mutant. The experiment was performed using three biological replicates, each with three technical replicates, and differences were statistically analyzed using Student's $t$-test ( ${ }^{* *} P<$ 0.01). Bars indicate the s.e.m

3: Table S2). Our findings demonstrated that the CRISPR/Cas9 system offers great potential in soybean breeding.

The circadian clock plays a critical role in the timing of multiple biological processes and stress responses in some model crops [16-18]. As key components of the circadian clock, LHY/CCA1 TFs have ability to initiate and set the phase of clock-controlled rhythms to produce a certain phenotype $[16,23,24,45,46]$. For example, the overexpression of $N a L H Y$ resulted in elongated hypocotyls and late flowering compared with WT plants in Nicotiana attenuata [23]. The same phenotypes were observed in Arabidopsis At $L H Y$-overexpressing lines [45, 46]. Although the functions of LHY/CCA1 were shown to be involved in flowering and stress responses in model crops, little is known regarding the biological functions of LHY/ CCA1 family members in soybean. To explore the molecular function of genes in soybean, we examined the phenotype of the loss-of-function of GmLHY in the $\mathrm{T}_{2}$ transgene-free mutant. We found that the plant height in the Gmlhy1a1b2a2b mutant was shortened in soybean at 20 to 35 DAE (Fig. 4A-E). Our data demonstrated that the clock gene GmLHY4, as an MYB TF, functions in regulating plant height in soybean.

Plant height is generally considered to be a central yield trait for breeding in various crops [3-5]. GAs is a large group of tetracyclic diterpenoid plant hormones that regulate diverse biological processes in plant growth and development, such as embryogenesis, leaf primordia, flowering, and plant height [47-49]. In recent years, a few GA metabolic pathway-related genes associated with plant height have been reported in plants [13, 14]. For example, $S D 1$ encodes a gibberellin 20-oxidase gene (GA20oxs), and the reduced endogenous GA levels in the $s d 1$ mutant led to the short stature of rice variety IR8 $[49,50]$. However, research on the molecular mechanisms of plant height regulation by TFs in soybean is lacking. In this study, the levels of endogenous GA3 in Gmlhy1a1b2a2b were lower than in WT, and the shortened internode phenotype could be rescued by treatment with exogenous GA3 (Fig. 5a-c). In addition, we tested the expression levels of GA synthetic genes (GmDW1, GmGA1, GmGA2, and GmCPS2) and GA response-related genes ( $G m G R 2$ and GmGR8) in the quadruple mutant of GmLHY and WT soybean plants (Fig. 6a-f). We found that these genes had substantially decreased expression in the quadruple mutant of GmLHY. Overall, we speculated that GmLHY might positively regulate the expression of these GA metabolic pathway-related genes to reduce soybean plant height.

\section{Conclusions}

The CRISPR/Cas9 system can be used for multiplex gene editing to advance crop plant breeding. In the present study, we used CRISPR/Cas9-based multiple genome editing to successfully obtain a quadruple mutant of $G m L H Y$ in soybean. Further, our results suggested that GmLHY directly or indirectly improves the expression level of GA synthetic genes and GA response-related genes to regulate soybean plant height. Our findings offer a case study for the use of gene editing to generate non-transgenic soybean genotypes and provide insight into the mechanisms underlying plant height regulatory networks in crop plant species.

\section{Methods}

\section{Plasmid construction}

The nucleotide sequences of the four GmLHY genes were downloaded from Phytozome (https://phytozome. 

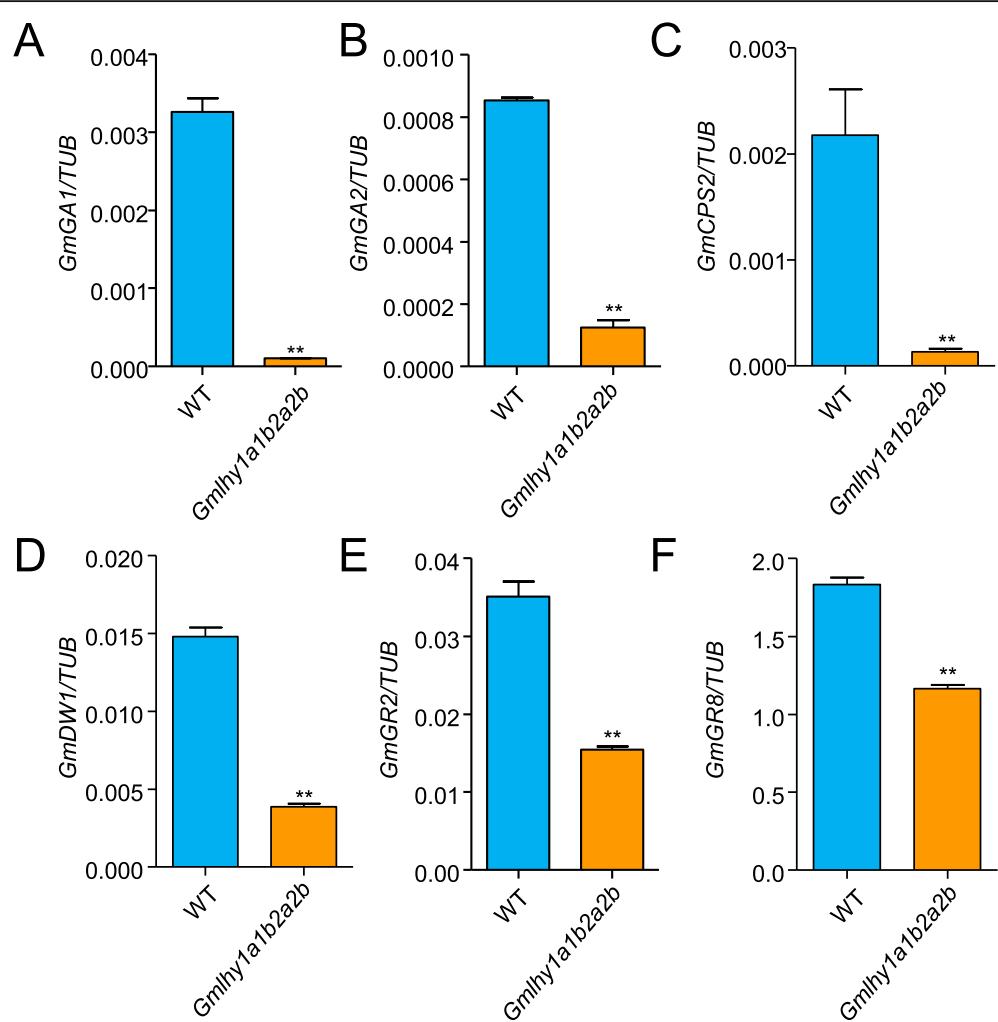

Fig. 6 The relative expression of GA metabolic pathway-related genes in the quadruple mutant of GmHY and WT soybean plants. a-d. The expression level of GA biosynthesisrelated genes; e-f The expression level of GA response-related genes. Soybean GmTUB was used as an internal control to normalize all data. The experiment was performed using three biological replicates, and differences were statistically analyzed using Student's $t$-test $\left({ }^{* *} P<0.01\right)$. Bars indicate the s.e.m.

jgi.doe.gov/pz/portal.html). The target sequences of the GmLHY genes were designed using the web tool CRISPR-P (http://cbi.hzau.edu.cn/crispr/). The pYLCRISPR/ Cas9P35S-B vector was a gift from Ma et al. [51]. The target sequences were subcloned into the different single guide RNA (sgRNA) expression cassettes and built into the pYLCRISPR/ Cas9P35S-B vector according to the protocol reported by Ma et al. [51]. The positive plasmids were introduced into Agrobacterium tumefaciens strain EHA101 for soybean stable transformation and into Agrobacterium rhizogenes strain K599 for soybean hairy roots transformation.

\section{Stable soybean transformation}

The transformation procedure was according to a previous protocol $[52,53]$. Putative transgenic soybean plants were screened by herbicide leaf-painting of $\mathrm{T}_{0}$ generation leaves at three vegetative stages (V3, V4, and V5) by wiping $100 \mathrm{mg} / \mathrm{L}^{-1}$ glufosinate-ammonium solution onto the upper leaf surface. Genomic DNA was extracted from the leaves of herbicide-resistant plants using a NuClean Plant Genomic DNA Kit (CWBIO, China). To confirm the presence of the Cas9 gene, PCR analysis was performed using Cas 9 gene-specific primers
(Additional file 4: Table S3). The PCR amplifications were performed once for each DNA sample.

\section{Agrobacterium rhizogenes-mediated transformation of soybean hairy roots}

Transgenic soybean hairy roots were generated by $A$. rhizogenes-mediated transformation as described by Kereszt et al. [40] and Cheng et al. [54] with some modifications. The cotyledons were cut into rough triangles and immediately placed into Petri dishes containing 0.8\% agar medium to keep them moist. The cut surface was treated with $20 \mu \mathrm{L}$ A. rhizogenes suspension. The dishes were sealed with Parafilm and placed in an incubator at $25^{\circ} \mathrm{C}$. Transformed hairy roots were abundant along a callus ridge on the inoculated cotyledons after approximately 2 weeks. The transgenic hairy roots were tested via PCR sequencing analysis.

\section{Identification of induced mutations using PCR and sequencing analyses}

DNA was isolated from the transgenic soybean hairy roots and transgenic plants using a NuClean Plant Genomic DNA Kit (CWBIO, China). The regions spanning the targets of the $G m L H Y$ genes were amplified using 


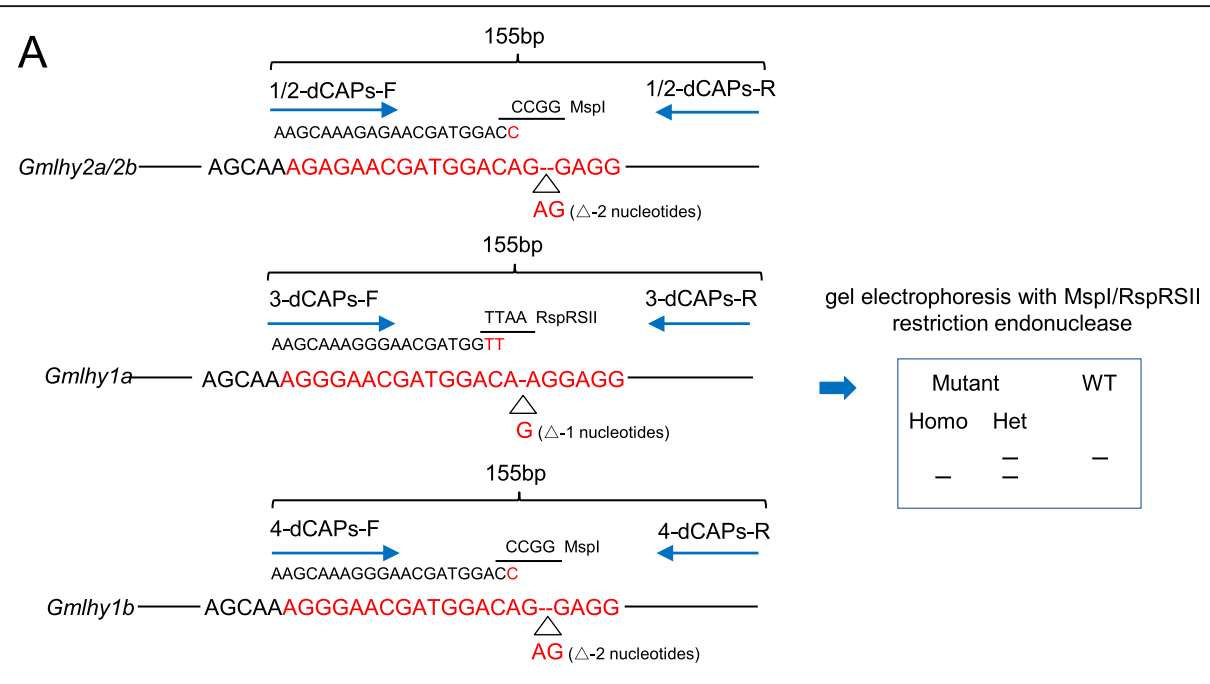

B

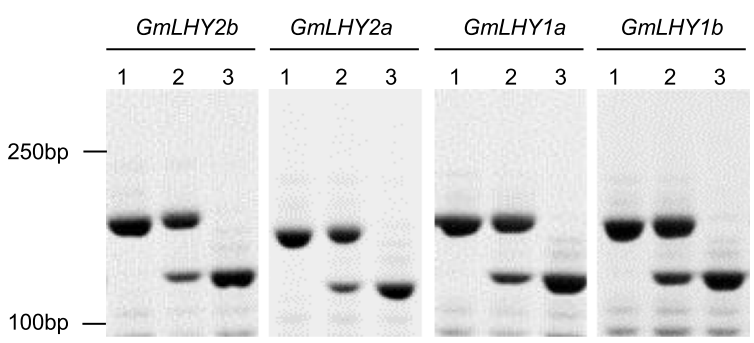

Fig. 7 Inheritance and segregation of GmLHY gene small deletions. a An example of the dCAPs-specific primer designed for quadruple mutant of GmLHY is shown; $\mathbf{b}$ The genotyping of the quadruple mutant of GmLHY is shown. "1" indicates no gene change. "2" indicates that the gene was heterozygous. "3" indicates that the gene was homozygous

KOD DNA Polymerase (Toyobo, Japan) with the different primer pairs in Additional file 4: Table S3. The sequences of the $\mathrm{T}_{0}$ and $\mathrm{T}_{1}$ generation plants and soybean hairy roots were analyzed using BioEdit to characterize the mutations induced by CRISPR/Cas9.

\section{Plant material, growth conditions, and primers}

The soybean cultivar 'Harosoy' was used for soybean hairy root and stable transformations. To investigate the plant height of the transgenic plants, the $T_{2}$ transgenefree mutants and WT control plants were grown in a growth chamber maintained at $25^{\circ} \mathrm{C}$ and $70 \%$ relative humidity with a $16 \mathrm{~h}$ light $/ 8 \mathrm{~h}$ dark cycle for 20-35 DAE. The node number on the main stem and internodal length were recorded at 20 DAE. The expression of GA biosynthesis genes and GA response-related genes was detected in the mutant and WT leaves at $20 \mathrm{DAE}$. All primers used for vector construction, PCR, and qRTPCR assays for all target genes are listed in Additional file 4: Table S3.

\section{qRT-PCR analysis}

Total RNA was isolated from the WT and $\mathrm{T}_{2}$ mutant soybean leaves using TRIzol reagent (Invitrogen, Shanghai, China). The cDNA synthesis was conducted using an M-MLV reverse transcriptase kit (Takara, Dalian, China) according to the manufacturer's instructions. The qRT-PCR analysis was used to measure the transcript levels of the $G m L H Y$ genes, namely GmGA1, GmGA2, GmCPS2, GmGR2, GmGR8, and $G m D W 1$, on a Roche LightCycler480 system (Roche, Germany) using a real-time PCR kit (Roche, Germany). The soybean housekeeping gene GmTubllin (Glyma.05G157300) was used as an internal reference to normalize all data. The relative transcript level of the target gene was calculated using the $2^{-\Delta \Delta C T}$ method. Three biological replications per line were performed in each test.

\section{Molecular marker development}

GmLHY sequences of the Harosoy and mutant genome were obtained by sequencing. Primers were designed using Primer Premier 5.0, with a product size $<200 \mathrm{bp}$. Three dCAPs markers were developed on the basis of the variations in the target 1/3 site of the GmLHY genes. $G m L H Y 2 a$ and $G m L H Y 2 b$ shared a pair of markers, and GmLHY1 $a$ and GmLHY1b each shared a pair of markers. Additional file 4: Table S3 lists the dCAPs markers that were used in this study. 


\section{$\mathrm{GA}_{3}$ and Uni treatment, and endogenous $\mathrm{GA}$ determination}

The Gmlhy1a1b2a2b mutant and WT were grown in a growth chamber at $25^{\circ} \mathrm{C}$ under LD (16 h light $/ 8 \mathrm{~h}$ dark) conditions, and $75 \%$ humidity. At approximately 20 DAE, $1 \mathrm{~g}$ (fresh weight) leaves tissue from the mutant or WT seedlings was harvested, weighed, immediately frozen in liquid nitrogen, and then stored at $-80^{\circ} \mathrm{C}$. The quantitative profiling of $\mathrm{GA}_{3}$ was determined using LCMS. These analyses were conducted by the Suzhou Comin Biotechnology (Suzhou, China).

To assess the response of the Gmlhy1a1b2a2b mutant to $\mathrm{GA}_{3}, 1.0 \mathrm{mg} / \mathrm{L}$ of $\mathrm{GA}_{3}$ was applied two times to seedlings with fully-open true leaves. The Uni $(1.0 \mathrm{mg} / \mathrm{L})$ treatment was carried out at the same time. The soybean growth condition was set as mentioned above. Three repeats were prepared for each treatment, and the effect of the hormone on stem expansion was evaluated $4 \mathrm{~d}$ later by measuring seedling length.

\section{Supplementary information}

Supplementary information accompanies this paper at https://doi.org/10. 1186/s12870-019-2145-8.

Additional file 1: Figure S1. Phylogenetic tree of $L H Y$ and CCA1 from Arabidopsis and soybean. The phylogenetic tree was inferred using the neighbor-joining method. The bootstrap consensus tree generated from 1000 replicates was used to represent the history of the different LHY/ CCA1 proteins analyzed. Figure S2. CRISPR/Cas9-induced mutations of the four GmLHY genes in transgenic soybean hairy roots. A. Growth of transgenic hairy roots in the culture medium for $14 \mathrm{~d}$. The typical lines were selected. B. Gel electrophoresis of PCR amplicons using specific primers for the CRISPR/Cas9 vector. C. Detailed sequence of the targets site in the transgenic soybean hairy roots. The red frames indicate the location of the targets. Figure S3. Sequencing of the CRISPR/Cas9-edited sites of $G m L H Y$ in the $T_{0}-7$ line. A. Gel electrophoresis of the PCR amplicons using specific primers for CRISPR/Cas9 vector. B-E. The fragments containing the edited sites were amplified by PCR and directly sequenced. The sequencing chromatograms with superimposed peaks derived from biallelic mutations of the targeted sites were decoded by the DSD ecode program [51]. The red frames indicate the location of the targets.

Additional file 2: Table S1. CRISPR/Cas9-meditated targeted mutagenesis of four GmLHY genes in transgenic soybean hairy roots.

Additional file 3: Table S2. CRISPR/Cas9-meditated targeted mutagenesis of four GmLHY genes in transgenic soybean plants.

Additional file 4: Table S3. Primers used for PCR and $q R T-P C R$ in this study.

\footnotetext{
Abbreviations

ABA: Abscisic acid; Br2: Brachytic2; Cas9: CRISPR-associated system 9; CPS2: Copalyl pyrophosphate synthase; CRISPR: Clustered regularly interspaced short palindromic repeat; DAE: Days after emergence; dCAPs: Derived cleaved amplified polymorphic sequences; DW1: Dwarf mutant; EE: Evening element; GA: Gibberellic acid; GA1/2: GA-20 oxidase1/2; GA3ox2: GA3 b-hydroxylase; GR2/8: GA-responsive gene 2/8; LC-MS: Liquid chromatography-mass spectrometry; LD: Long-day; LHY: LATE ELONGATED HYPOCOTYL; qRT-PCR: quantitative real-time PCR; TF: Transcription factor; TOC1: TIMING OF CAB EXPRESSION 1; WT: Wild type
}

\section{Acknowledgements}

We thank Professor Yaoguang Liu at the South China Agricultural University for providing the vector pYLCRISPR/Cas9P35S. We thank LetPub (www. letpub.com) for its linguistic assistance during the preparation of this manuscript.

\section{Authors' contributions}

FK and BL designed the experiments and managed the projects. QC, LD and TS performed experiments. TL modified and revised the manuscript. $Z G, H N$, $\mathrm{SL}, \mathrm{CF}, \mathrm{LK}, \mathrm{HY}, \mathrm{ZH}, \mathrm{KK}, \mathrm{YT}, \mathrm{XL}, \mathrm{XZ}$ and LC performed data analysis. FK, QC and LD wrote the manuscript.

\section{Funding}

This work was supported by National Natural Science Foundation of China (31901568, 31725021, 31771815, 31701445, 31801384). This work was also funded by the Natural Key R\&D Program of China (2017YFE0111000 and 2016YFD0100400). The funding body had no role in the design of the study and collection, analysis, and interpretation of data and in writing the manuscript.

\section{Availability of data and materials}

The datasets and materials developed and analyzed in this study are available from the corresponding author on reasonable request.

Ethics approval and consent to participate

Not applicable.

\section{Consent for publication}

Not applicable.

\section{Competing interests}

The authors have no competing interests to declare.

\section{Author details}

${ }^{1}$ School of Life Sciences, Guangzhou University, Guangzhou, China. ${ }^{2}$ The Innovative Academy of Seed Design, Key Laboratory of Soybean Molecular Design Breeding, Northeast Institute of Geography and Agroecology,

Chinese Academy of Sciences, Harbin, China. ${ }^{3}$ University of Chinese Academy of Sciences, Beijing, China.

Received: 20 August 2019 Accepted: 18 November 2019

Published online: 18 December 2019

\section{References}

1. Hartung RC, Specht JE, Williams JH. Modification of soybean plant architecture by genes for stem growth habit and maturity. Crop Sci. 1981; 21:51-6.

2. Huyghe C. Genetics and genetic modifications of plant architecture in grain legumes: a review. Agronomie. 1998;18:383-411.

3. Cooper RL, Martin RJ, St. Martin SK, Calip-DuBois A, Fioritto RJ, Schmitthenner AF. Registration of 'Charleston' soybean. Crop Sci. 1995; 35:593.

4. Cooper RL, Mendiola T. St. Martin SK, Fioritto RJ, Dorrance AE. Registration of 'apex' soybean. Crop Sci. 2003;43:1563.

5. Reinhardt D, Kuhlemeier C. Plant architecture. EMBO Rep. 2002;3:846-51.

6. Wang $H$, Nussbaum-Wagler T, Li B, Zhao Q, Vigouroux Y, Faller M, et al. The origin of the naked grains of maize. Nature. 2005:436:714-9.

7. Teng F, Zhai LH, Liu RX, Bai W, Wang LQ, Huo DG, et al. ZmGA3ox2, a candidate gene for a major QTL, QPH3.1, for plant height in maize. Plant J. 2013;73:405-16

8. Xing AQ, Gao YF, Ye LF, Zhang WP, Cai LC, Ching A, et al. A rare SNP mutation in Brachytic2 moderately reduces plant height and increases yield potential in maize. J Exp Bot. 2015;66:3791-802.

9. Yano M, Katayose Y, Ashikari M, Yamanouchi U, Monna L, Fuse T, et al. Hd1, a major photoperiod sensitivity quantitative trait locus in rice, is closely related to the Arabidopsis flowering time gene CONSTANS. Plant Cell. 2000; 12:2473-83

10. Fan C, Xing Y, Mao H, Lu T, Han B, Xu C, et al. GS3, a major QTL for grain length and weight and minor QTL for grain width and thickness in rice, encodes a putative transmembrane protein. Theor Appl Genet. 2006;112: 1164-71. 
11. Xue W, Xing $Y$, Weng $X$, Zhao $Y$, Tang $W$, Wang $L$, et al. Natural variation in Ghd7 is an important regulator of heading date and yield potential in rice. Nat Genet. 2008;40:761-7.

12. Frary A, Nesbitt TC, Grandillo S, Knaap E, Cong B, Liu J, et al. fw2.2: a quantitative trait locus key to the evolution of tomato fruit size. Science. 2000;289:85-8.

13. Li ZF, Guo Y, Ou L, Hong HL, Wang J, Liu ZX, et al. Identification of the dwarf gene GmDW1 in soybean (Glycine max L.) by combining mapping-bysequencing and linkage analysis. Theor Appl Genet. 2018;131:1001-16.

14. Jing $Y$, Zhao X, Wang J, Lian M, Teng WL, Qiu LJ, et al. Identification of loci and candidate genes for plant height in soybean (Glycine max) via genomewide association study. Plant Breed. 2019;00:1-12.

15. Chen X, Lu SC, Wang YF, Zhang X, Lv B, Luo LQ, et al. OsNAC2 encoding a NAC transcription factor that affects plant height through mediating the gibberellic acid pathway in rice. Plant J. 2015;82:302-14.

16. Nagel DH, Doherty CJ, Pruneda-Paz JL, Schmitz RJ, Ecker JR, Kay SA. Genome-wide identification of CCA1 targets uncovers an expanded clock network in Arabidopsis. Proc Nat Acad Sci. 2015;112(34):E4802-10.

17. Michael TP, Mockler TC, Breton G, McEntee C, Byer A, Trout JD, et al. Network discovery pipeline elucidates conserved time-of-day-specific cisregulatory modules. PLoS Genet. 2008:4(2):e14

18. Filichkin SA, Breton G, Priest HD, Dharmawardhana P, Jaiswal P, Fox SE, et al. Global profiling of rice and poplar transcriptomes highlights key conserved circadiancontrolled pathways and cis-regulatory modules. PLoS One. 2011;6(6):e16907.

19. Genoud T, Millar AJ, Nishizawa N, Kay SA, Schafer E, Nagatani A, et al. An Arabidopsis mutant hypersensitive to red and far-red light signals. Plant Cell. 1998;10:889-904.

20. Wang ZY, Tobin EM. Constitutive expression of the CIRCADIAN CLOCK ASSOCIATED (CCA1) gene disrupts circadian rhythms and suppresses its own expression. Cell. 1998:93:1207-17.

21. Alabadí D, Oyama T, Yanovsky MJ, Harmon FG, Más P, Kay SA. Reciprocal regulation between TOC1 and LHY/CCA1 within the Arabidopsis circadian clock. Science. 2001;293(5531):880-3.

22. Nakamichi N, Kiba T, Henriques R, Mizuno T, Chua NH, Sakakibara H. PSEUDO-RESPONSE REGULATORS 9, 7, and 5 are transcriptional repressors in the Arabidopsis circadian clock. Plant Cell. 2010;22:594-605.

23. Yon F, Joo Y, Cortés Llorca L, Rothe E, Baldwin IT, Kim SG. Silencing Nicotiana attenuata LHY and ZTL alters circadian rhythms in flowers. New Phytol. 2016;209(3):1058-66.

24. Adams S, Grundy J, Veflingstad SR, Dyer NP, Hannah MA, Ott S, et al. Circadian control of abscisic acid biosynthesis and signaling pathways revealed by genome-wide analysis of LHY binding targets. New Phytol. 2018;220(3):893-907.

25. Li JF, Norville JE, Aach J, McCormack M, Zhang DD, Bush J, et al. Multiplex and homologous recombination-mediated genome editing in Arabidopsis and Nicotiana benthamiana using guide RNA and Cas9. Nat Bio technol. 2013:31:688-91.

26. Ricroch A, Clairand P, Harwood W. Use of CRISPR systems in plant genome editing: toward new opportunities in agriculture. Emerg Top Life Sci. 2017;1:169-82.

27. Shan QW, Wang YP, Li J, Zhang Y, Chen KL, Liang Z, et al. Targeted genome modification of crop plants using a CRISPR Cas system. Nat Bio technol. 2013;31:686-8

28. Liu X, Wu S, Xu J, Sui C, Wei J. Application of CRISPR/Cas9 in plant biology. Acta Pharm Sin B. 2017;7(3):292-302.

29. YW, Bai Y, Wu GH, Zou SH, Chen YF, Gao CX, et al. Simultaneous modification of three homoeologs of TaEDR1 by genome editing enhances powdery mildew resistance in wheat. Plant J. 2017; 91:714-24.

30. Zhang SJ, Zhang RZ, Song GQ, Gao J, Li W, Han XD, et al. Targeted mutagenesis using the agrobacterium tumefaciens-mediated CRISPR-Cas9 system in common wheat. BMC Plant Biol. 2018;18:302.

31. Liang Z, Zhang K, Chen KL, Gao CX. Targeted mutagenesis in zea mays using TALENs and the CRISPR/Cas system. J Genet Genomics. 2014;41:63-8.

32. Shi JR, Gao HR, Wang HY, Lafitte HR, Archibald RL, Yang MZ, et al. ARGOS8 variants generated by CRISPR-Cas9 improve maize grain yield under field drought stress conditions. Plant Bio technol J. 2017;15:207-16.

33. Liu YF, Zhang DJ, Ping JQ, Li S, Chen ZX, Ma JX. Innovation of a regulatory mechanism modulating semi-determinate stem growth through artificial selection in soybean. PLoS Genet. 2016;12:e1005818.

34. Lawrenson T, Shorinola O, Stacey N, Li CD, Ostergaard L, Patron N, et al. Induction of targeted, heritable mutations in barley and Brassica oleracea using RNA-guided Cas9 nuclease. BMC Genome Biol. 2015;16:258.
35. Soyk S, Muller NA, Park SJ, Schmalenbach I, Jiang K, Hayama R, et al. Variation in the flowering gene SELF PRUNING 5G promotes day-neutrality and early yield in tomato. Nat Genet. 2017;49:162-8.

36. Soyk S, Lemmon ZH, Oved M, Fisher J, Liberatore KL, Park SJ, et al. Bypassing negative epistasis on yield in tomato imposed by a domestication gene. Cell. 2017;04:032

37. Li ZS, Liu ZB, Xing AQ, Moon BP, Koellhoffer JP, Huang LX, et al. Cas9-guide RNA directed genome editing in soybean. Plant Physiol. 2015;169:960-70.

38. Bao A, Chen H, Chen L, Chen S, Hao Q, Guo W, et al. CRISPR/Cas9-mediated targeted mutagenesis of GmSPL9 genes alters plant architecture in soybean. BMC Plant Biol. 2019:19(1):131.

39. Do PT, Nguyen CX, Bui HT, Tran LTN, Stacey G, Gillman JD, et al. Demonstration of highly efficient dual gRNA CRISPR/Cas9 editing of the homeologous GMFAD2-1A and GMFAD2-1B genes to yield a high oleic, low linoleic anda-linolenic acid phenotype in soybean. BMC Plant Biol. 2019:19:311.

40. Kereszt A, Li D, Indrasumunar A, Nguyen CD, Nontachaiyapoom S, Kinkema $\mathrm{M}$, et al. Agrobacterium rhizogenes-mediated transformation of soybean to study root biology. Nat Protoc. 2007;2:948-52.

41. Helliwell CA, Sheldon CC, Olive MR, Walker AR, Zeevaart JA, Peacock WJ, et al. Cloning of the Arabidopsis ent-kaurene oxidase gene GA3. Proc Natl Acad Sci. 1998;95:9019-24.

42. Ji S, Gururani M, Lee J, Ahn B, Chun S. Isolation and characterization of a dwarf rice mutant exhibiting defective gibberellins biosynthesis. Plant Biol. 2014;16:428-39.

43. Michno JM, Wang X, Liu J, Curtin SJ, Kono TJ, Stupar RM. CRISPR/Cas mutagenesis of soybean and Medicago truncatula using a new web-tool and a modified Cas9 enzyme. GM Crops Food. 2015;6:243-52.

44. Kanazashi Y, Hirose A, Takahashi I, Mikami M, Endo M, Hirose S, et al. Simultaneous site-directed mutagenesis of duplicated loci in soybean using a single guide RNA. Plant Cell Rep. 2018;37:553-63.

45. Schaffer R, Ramsay N, Samach A, Corden S, Putterill J, Carre IA, et al. The late elongated hypocotyl mutation of Arabidopsis disrupts circadian rhythms and the photoperiodic control of flowering. Cell. 1998:93:1219-29.

46. Somers DE, Kim WY, Geng R. The F-box protein ZEITLUPE confers dosagedependent control on the circadian clock, photomorphogenesis, and flowering time. Plant Cell. 2004;16:769-82.

47. Kaneko M, Itoh H, Inukai Y, Sakamoto T, Ueguchi-Tanaka M, Ashikari M, et al. Where do gibberellin biosynthesis and gibberellin signaling occur in rice plants? Plant J. 2003;35:104-15.

48. Sun T, Gubler F. Molecular mechanism of gibberellin signaling in plants. Annu Rev Plant Biol. 2004;55:197-223.

49. Sasaki A, Ashikari M, Ueguchi-Tanaka M, Itoh H, Nishimura A, Swapan D, et al. A mutant gibberellins-synthesis gene in rice. Nature. 2002;416:701-2.

50. Spielmeyer W, Ellis MH, Chandler PM. Semi dwarf (sd-1), "green revolution" rice, contains a defective gibberellin 20-oxidase gene. Proc Natl Acad Sci. 2002;99:9043-8.

51. Ma XL, Zhang QY, Zhu QL, Liu W, Chen Y, Qiu R, et al. A robust CRISPR/Cas9 system for convenient, high-efficiency multiplex genome editing in monocot and dicot plants. Mol Plant. 2015;8:1274-84.

52. Zeng P, Vadnais DA, Zhang Z, Polacco JC. Refined glufosinate selection in agrobacterium-mediated transformation of soybean [Glycine max (L.) Merrill]. Plant Cell Rep. 2004; 22:478-82.

53. Flores T, Karpova O, Su X, Zeng P, Bilyeu K, Sleper DA, et al. Silencing of GMFAD3 gene by siRNA leads to low alpha-linolenic acids (18:3) of fad3mutant phenotype in soybean [Glycine max (Merr.)]. Transgenic Res. 2008;17: 839-50

54. Cheng Q, Dong LD, Gao TJ, Liu TF, Li NH, Wang L, et al. The bHLH transcription factor GmPIB1 facilitates resistance to Phytophthora sojae in Glycine max. J Exp Bot. 2018;69:2527-41.

\section{Publisher's Note}

Springer Nature remains neutral with regard to jurisdictional claims in published maps and institutional affiliations. 